\title{
Los procesos de transición democrática en los países latinoamericanos a través de las publicaciones españolas recogidas en la base de datos "América Latina" del CINDOC"
}

\author{
Adelaida Román Román \\ y Graciela Fainstein Lamuedra \\ CINDOC, CSIC, Madrid
}

A partir de los elementos bibliográficos de las publicaciones españolas sobre la transición a la democracia en los países latinoamericanos, editadas entre 1980 y 1995, se analizan los aspectos y los países que interesaron más a los autores, se aportan las características que ofrece la producción bibliográfica sobre el tema y se estudian los elementos comunes y diferenciales con el proceso de transición español, considerado éste también desde la producción bibliográfica generada.

\section{Introducción}

Desde hace unos veinte años hasta hoy, se han realizado numerosos estudios, analíticos unos, generalizadores otros, sobre los procesos de transición política en España, en los países latinoamericanos y más recientemente en los países del Este de Europa. Pero no son muchos hasta ahora los trabajos que han abordado el análisis de las características que presenta esta producción bibliográfica ni en su conjunto, ni para cada país.

El propósito de este trabajo es aproximarse a la bibliografía publicada en España sobre las transiciones políticas en los países latinoamericanos, utilizando los elementos que aportan los registros bibliográficos, para caracterizar tanto los aspectos más formales de esa producción bibliográfica como aquellos relacionados con los temas estudiados, su alcance geográfico, su dimensión institucional, los vehículos de difusión utilizados, etc.

¿Qué puede aportar al conocimiento de los procesos de transición a la democracia tal y como han tenido lugar en América Latina un estudio de estas características? ¿puede establecerse algún tipo de relación entre los

* Este trabajo fue presentado como comunicación en el V Encuentro de Latinoamericanistas españoles, celebrado en Sevilla, en noviembre de 1995. La bibliografía que ha sido utilizada para elaborar esta comunicación, está disponible en Cuadernos Rayuela, N. 7 , de 1995: "Transiciones a la democracia en América Latina", editado por el CINDOC. 
estudios publicados sobre un tema y su representación en una Base de Datos, en forma de registros bibliográficos? ¿No hay demasiadas mediaciones para poder sacar conclusiones creibles?

El objeto de esta ponencia no es tanto decir cosas sobre las transiciones políticas sino más bien sobre los estudios acerca de esas transiciones y el modo como los autores se han aproximado a ellas.

\section{Fuentes y metodología}

El material de trabajo utilizado para esta comunicación se ha extraído de la Base de Datos "América Latina", del CINDOC, actualizada a 20 de septiembre de 1995. Esta base de datos recoge, desde 1975, todos los trabajos publicados en revistas españolas, así como los capítulos de libros (compilaciones u obras colectivas), ponencias y comunicaciones a congresos, tesis e informes. Solamente quedan excluidas las monografías. ${ }^{1}$

Se han obtenido de la Base de Datos todos los registros correspondientes a los trabajos publicados desde 1980 hasta julio de 1995. El resultado ha sido un conjunto de 211 documentos que responden a la siguiente ecuación de búsqueda: ((Transición política o cambio político) y democra* $^{*}$ o transición democrática. ${ }^{2}$

La única restricción ha sido la temporal ya mencionada. Temáticamente, se ha considerado que los trabajos centrados en el estudio de la consolidación de la democracia correspondían a una etapa posterior a las transiciones propiamente dichas y por ello no han sido incluidos.

La disponibilidad de esta información en forma electrónica nos ha facilitado la elaboración y la cuantificación de los datos. Por otra parte, el hecho de tener acceso físicamente a los ejemplares ha permitido garantizar la idoneidad de la descripción temática que se asigna en la base de datos a cada registro bibliográfico.

Para el análisis de los temas abordados en los trabajos se ha tenido en cuenta la frecuencia de aparición de las palabras clave que representan los contenidos fundamentales de cada documento, siguiendo el principio de

1 El término "monografías" es utilizado aquí como sinónimo de "libros", en los que la autoría, sea de uno o varios autores, afecta a toda la obra. Se diferencia así de las "compilaciones", libros fruto de la colaboración de varios autores, que son responsables de capítulos concretos, y no del conjunto de la obra.

2 El asterisco significa truncamiento, posibilidad que tienen los sistemas de recuperación de, mediante un operador sintáctico, el *, recuperar todos los términos que tienen la misma raíz. 
que la mayor o menor presencia de un término no vacío en un texto es un indicador de la mayor o menor centralidad del tema representado por dicho término en ese texto.

El trabajo abordará cuatro aspectos: a) Las características de la comunidad científica que escribe en publicaciones españolas sobre las transiciones latinoamericanas a la democracia, así como de la producción bibliográfica generada por esa comunidad científica. b) Descripción general de los contenidos de los trabajos publicados. c) Encuentros y desencuentros con lo publicado sobre la Transición española. d) Análisis del contenido de los trabajos sobre las transiciones en los países más estudiados.

\section{La comunidad científica}

\section{Los autores}

Los 219 trabajos recogidos sobre las transiciones a la democracia en América Latina han sido escritos por 174 autores. En la descripción de cualquier corpus bibliográfico un dato interesante a estudiar es siempre el comportamiento de éstos, y en concreto lo relativo a su productividad.

Lotka formuló en 1926 la Ley de Productividad de los Autores como "ley cuadrática inversa de la productividad" según la cual, el número de autores que producen "n" trabajos es proporcional a 1/n2. Esta ley es aplicable cuando la bibliografía estudiada reune dos condiciones: cubrir un período de tiempo suficientemente amplio y tratarse de un corpus bibliográfico de grandes dimensiones. Nuestra bibliografía cumpliría la primera condición pero quizás no suficientemente la segunda. Por esta razón hemos optado por estudiar la distribución de los trabajos por autor extrayendo de estos datos la productividad media de los autores.

Los 219 trabajos objeto de este estudio escritos por 174 autores, arrojan una media de 1,25 trabajos por autor, productividad algo más baja, pero muy cercana a la de los autores que publicaron en España sobre la Transición española entre 1975 y 1991, y bastante más baja que la media de los autores españoles de ciencias sociales, ${ }^{3}$ muy próxima a los valores ofrecidos por Kyvik ${ }^{4}$ para los científicos sociales de las universidades noruegas.

3 Román Román, A.: Las revistas de Ciencias Sociales, fuente para el estudio de la Historia. La Transición política (1975-1985). Una perspectiva historiográfica y documental. Madrid, 1993 (ed. en microficha).

4 Kyvik, Svein: Productivity in Academia. Oslo, 1991. 
El 75,4\% de los autores lo son sólo de un trabajo y el 24,5\% lo son de dos o más trabajos. Todos los autores se sitúan en el nivel de "pequeños productores", entendiendo por esto los autores que produjeron menos de 10 trabajos en el período estudiado. Sólo dos autores superan los 5, con 6 y 7 trabajos respectivamente.

La relación de firmas-trabajo es la siguiente:

Trabajos de un solo autor

Trabajos de dos autores

Trabajos de tres autores

Trabajos de cuatro autores
199

$90,9 \%$

$7,3 \%$

$0,4 \%$

$3 \quad 1,4 \%$

Así, el $91 \%$ de los trabajos es obra de un solo autor individual y sólo el 9\% restante es fruto de la colaboración entre dos o más autores. La media de firmas-trabajo se sitúa en un 1,12, muy por debajo del 2,5, media considerada como normal a nivel internacional, que revela lo poco consolidado que está aún en nuestro país el hábito del trabajo en equipo.

\section{Las instituciones en que trabajan los autores}

La afiliación institucional de los autores es un dato del máximo interés en cualquier estudio descriptivo de un corpus bibliográfico. Sin embargo, los autores españoles no acaban de incorporar el hábito de consignar este dato en sus publicaciones. De los 219 trabajos recogidos, sólo los autores de 107 de estos trabajos aportan este dato (49\%). Por tanto las consideraciones sobre los lugares de trabajo de los autores se referirán únicamente a ese $49 \%$. Estos son los datos:

Universidades ..................... 56\%

Instituciones de investigación y estudio $\ldots \ldots \ldots \ldots 27,4 \%$

Organismos internacionales $\ldots \ldots \ldots \ldots \ldots \ldots \ldots, 5 \%$

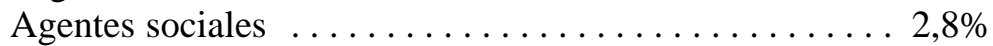

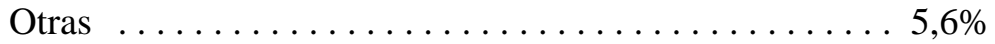

Como cabía esperar, son las instituciones dedicadas a la investigación - universidades, institutos de investigación, asociaciones científicas, etc.- las que producen la mayor parte de los trabajos publicados, un $84 \%$. 
Llama la atención la baja aportación de los agentes sociales (partidos políticos, sindicatos, movimientos de liberación, movimientos sociales, etc..) al estudio de un tema que les atañe tan directamente.

\section{Origen geográfico de los trabajos}

A pesar de que trabajamos con un corpus bibliográfico editado en España, es interesante pararse a considerar los lugares en los que se generaron los trabajos publicados. Por los datos que nos aporta ese $49 \%$ de documentos que consignan la afiliación institucional de los autores, podemos deducir la tendencia de las contribuciones que sobre las transiciones latinoamericanas han hecho los diferentes países a la producción española.

Atendiendo al lugar de trabajo de los autores, este es el orígen geográfico de los documentos: América Latina, el 49\%, desglosado en: Argentina, 5,0\%; Brasil, 5,6\%; Chile, 12,2\%; Colombia, 5,0\%; México, 8,5\%, y Uruguay, 5,0\%. España, el 34,5\%, desglosado entre: Barcelona, 7,5\%; La Coruña, 1,0\%; Madrid, 23,0\%, y Salamanca, 3,0\%. Estados Unidos, un $5,0 \%$. Europa, 7,5\%, e instituciones no localizadas, el 4,0\% restante.

Puede observarse que las aportaciones publicadas en nuestro país son, en primer lugar, debidas a autores de países latinoamericanos (49\%), entre los que destaca Chile. El segundo lugar lo ocupan los autores que trabajan en instituciones españolas (34,5\%), con una fuerte centralización en Madrid, y ausencia de regiones con tanta tradición americanista como Andalucía. Las aportaciones de otros países europeos y de los Estados Unidos ocupan el tercer y el cuarto lugar respectivamente.

Hay que reiterar que estos datos sólo pueden tomarse como indicativos de lo que ocurre, y es muy posible que la presencia tan dominante de autores extranjeros se deba a la mayor tradición entre éstos de cumplimiento de las normas internacionales de publicación de trabajos científicos que obligan a hacer constar la afiliación institucional de los autores.

\section{Características formales de la bibliografía estudiada}

Los documentos extraídos de la B.D. "América Latina" son fundamentalmente artículos de revista, pero hay un número nada desdeñable de otro tipo de documentos como tesis, ponencias a congresos, contribuciones 
a compilaciones, etc. La distribución por tipo de documentos es la siguiente: Artículos de revista, 128 (58,4\%); capítulos de compilaciones, 61 $(27,8 \%)$; ponencias, $22(10 \%)$; tesis, $7(3,2 \%)$, e informes, $1(0,5 \%)$.

En esta distribución se verifica una vez más la preferencia de los autores por las revistas como vehículo de difusión de su actividad científica, sin embargo es de destacar que los documentos que no son artículos de revista representan en este corpus más del $40 \%$ del total de trabajos, siempre excluyendo las monografías, poniendo de manifiesto la importancia que tiene para la investigación el que este tipo de documentos de más difícil acceso puedan ser recogidos e incluidos en las B.D. y estar así localizables y disponibles.

Las revistas que publicaron trabajos sobre la transición a la democracia en los países latinoamericanos fueron las siguientes:

Síntesis

Pensamiento iberoamericano

Revista de Estudios Políticos

América Latina Hoy

Tiempo de Paz

Leviatán

Papers

Revista Internacional de Ciencias Sociales

Revista Internacional de Sociología

Sistema

Claves de Razón Práctica

Contrarios

Cuenta y Razón

Papeles para la Paz

Situación Latinoamericana

17 títulos con un solo trabajo

$\begin{array}{rr}35 & 20,0 \% \\ 14 & 11,0 \% \\ 14 & 11,0 \% \\ 13 & 10,0 \% \\ 7 & 5,4 \% \\ 4 & 3,0 \% \\ 4 & 3,0 \% \\ 4 & 3,0 \% \\ 4 & 3,0 \% \\ 3 & 2,3 \% \\ 3 & 2,3 \% \\ 2 & 1,5 \% \\ 2 & 1,5 \% \\ 2 & 1,5 \% \\ 2 & 1,5 \% \\ & 13,3 \%\end{array}$

Si observamos la distribución de los artículos por revistas, veremos que únicamente el 50\% de ellos han sido publicados en revistas americanistas, el otro $50 \%$ ha visto la luz en revistas de ciencias sociales no especializadas en América Latina.

Este hecho, unido a la gran dispersión de los estudios publicados, da idea de la dificultad de reunir, en un momento dado, la información específica producida sobre un tema, pues obligaría a revisar un número grande de 
títulos. En sentido contrario, y para el tema de las transiciones a la democracia, los cuatro títulos más productivos concentran casi el $60 \%$ de los artículos publicados. Conviene hacer constar que Síntesis, que aparece como revista más productiva en relación con este tema, a mucha distancia del resto, dedicó un número monográfico, en 1994, sobre los procesos de democratización en América Latina.

Un último aspecto que interesa tratar en este punto es el de la distribución por años de la producción bibliográfica sobre las transiciones a la democracia. A continuación se consignan los trabajos por año de publicación, en orden de mayor a menor número de documentos sobre el tema: 1994, 35 (16\%); 1992, 30 (13,7\%); 1991, 28 (12,8\%); 1995, 25 (11,4\%); 1990, 24 (11\%); 1988, 17 (7,7\%); 1986, 16 (7,3\%); 1993, 11 (5\%); 1987, 9 (4,1\%); 1989, 7 (3,2\%); 1982, 7 (3,2\%); 1983, 6 (2,7\%); 1984, 3 (1,4\%); 1981, $2(0,9 \%)$, y 1985 , con $1(0,45 \%)$.

Parece evidente que la mayor cantidad de trabajos publicados lo ha sido en los últimos cinco años, siempre excluyendo las monografías, no contempladas en esta comunicación. Este hecho se ve incluso reforzado debido a que la literatura producida en 1995 aún no está disponible en su totalidad, y cabe suponer que desde septiembre, fecha de la actualización de la B.D., hasta que el año termine, podrán seguir incorporándose otros documentos que se vayan publicando sobre el tema.

\section{Contenido de los trabajos publicados en España sobre las transiciones a la democracia en América Latina}

Basaremos el estudio de los temas tratados en el análisis de los descriptores o palabras clave que acompañan a los registros bibliográficos en la B.D. "América Latina" y que son resultado del análisis de contenido realizado sistemáticamente por los analistas documentales que alimentan la B.D.

Una vez obtenida la relación alfabética de los descriptores con su frecuencia de aparición en este corpus, y con el objeto de ofrecer primero una visión global de los contenidos dominantes, se procede a categorizar los documentos, incluyéndolos en una de estas tres categorías: descriptores del ámbito económico "E", descriptores del ámbito jurídico-político "JP", y descriptores del ámbito socio-cultural "SC". Se establece un cuarto grupo 
para incluir en ellos los términos neutros desde el punto de vista de su contenido conceptual (evolución, método, análisis...) "N".

Aportamos a continuación los parámetros cuantitativos del conjunto estudiado:

Número de documentos . . . . . . . . . . . . . . . . 219

Número de descriptores únicos . . . . . . . . . . . . 434

Descriptores asignados a los documentos . . . . . . 1.834

Media de descriptores por documento $\ldots \ldots \ldots \ldots \ldots 8,4^{5}$

Para el recuento de los descriptores por categorías, eliminamos aquellos que sirvieron para establecer la ecuación de búsqueda ya que, lógicamente, se encuentran presentes (una combinación de ellos) en todos los documentos del corpus, y por tanto su presencia no es significativa. Fueron la condición de la obtención de la bibliografía: transición política, democracia, democratización, cambio político, transición democrática.

Los datos del recuento de descriptores y sus frecuencias, una vez categorizados, son los siguientes:

$\begin{array}{lrr}\text { Descriptores "E" } & 268 & 14,6 \% \\ \text { Descriptores "SC" } & 233 & 12,7 \% \\ \text { Descriptores "JP" } & 848 & 46,2 \% \\ \text { Términos "N" } & 26 & 1,4 \%\end{array}$

Por los datos puede verse que desde el punto de vista de la temática general, más de un $46 \%$ de los documentos abordan los aspectos políticojurídicos de las transiciones, y que los problemas de órden económico, social y cultural han sido estudiados en bastante menor medida.

Para un análisis un poco más fino de los temas estudiados, hemos tenido en cuenta aquellos términos que aparecen al menos en 5 documentos. Es obvio que a mayor frecuencia de aparición menor número de términos (ley de Zipf). ${ }^{6}$ Queremos decir con esto que los términos con frecuencia de aparición menor que cinco son mucho más numerosos, pero menos significa-

5 La suma de porcentajes no representa el $100 \%$ del total, ya que no se han contabilizado los términos empleados para realizar la búsqueda bibliográfica en la B.D. para no sesgar.

6 Zipf, George K.: Selected studies of the principle of relative frequency in language. Cambridge, Mass., 1932. 
tivos. Queremos vincular así la representatividad de los términos en el corpus bibliográfico a su frecuencia de aparición.

\section{Aspectos económicos más tratados}

Los descriptores "E" con frecuencia superior a 5 son doce, con un total de 120 ocurrencias. El aspecto más presente es la crisis económica, de la que hablan 31 documentos. Siguen en interés la política económica, los temas de cooperación al desarrollo y de los modelos de desarrollo, los problemas derivados de la deuda externa, y todo lo relativo a los procesos de integración regional. Cada uno de estos aspectos está presente en el corpus a un nivel equivalente. En un segundo nivel aparecen temas como la reforma económica, las políticas de ajuste, las políticas neoliberales, el papel del Estado en este nuevo modelo neoliberal, y también los problemas planteados por la situación de países económicamente dependientes.

\section{Aspectos socioculturales}

Estos son, sin duda, los que menos interés suscitan a los investigadores, al menos a los que publican en nuestro país trabajos sobre la transición a la democracia. Sólo superan las cinco ocurrencias un pequeño conjunto de términos, 8, con un total de 71 frecuencias. Los aspectos más estudiados son los relativos a los movimientos sociales. En un segundo nivel, modernización, sindicatos, cambio social, sociedad civil y pobreza, son los temas que han interesado más.

\section{Aspectos jurídico-políticos}

Este conjunto es con mucho el más grande. Está formado por 51 descriptores que tienen más de cinco ocurrencias con un total de 599 frecuencias, sin contar las sumadas por los términos de búsqueda que todos pertenecen a este conjunto.

Se establecen tres niveles de importancia en los temas tratados de acuerdo con su frecuencia de aparición. Un primer nivel estará formado por los temas que están presentes en más de 20 documentos, un segundo nivel 
será el de aquellos temas tratados en más de 10 y menos de 21 , y un tercer nivel de interés estará representado por los temas que aparecen en menos de 10 documentos.

Siguiendo este esquema, el papel de los partidos políticos en los procesos de democratización junto con todo lo relativo a los procesos electorales han sido los aspectos que más interés han suscitado entre los autores. Los regímenes de dictadura y en especial las dictaduras militares, los sistemas políticos, los problemas de consolidación democrática estudiados conjuntamente con los procesos de transición, los problemas relacionados con las Fuerzas Armadas, y las relaciones internacionales completan el conjunto de aspectos estudiados que situaríamos en un primer nivel de interés.

En un segundo nivel, encontramos trabajos que analizan rasgos del ejercicio del poder político como el autoritarismo, o el presidencialismo, estudios centrados en los diversos aspectos de la participación política como las fuerzas políticas, la oposición política, las limitaciones de la democracia formal, las crisis políticas, las garantías para ejercer los derechos civiles, o la incidencia de la revolución armada en los procesos de cambio político en Centroamérica.

Un tercer grupo estaría formado por los artículos que abordan aspectos vinculados al desarrollo político, desarrollo constitucional, cultura política, consenso político, representación política, etc., y otros relacionados con la legitimidad política, la estabilidad, o las opciones políticas, izquierda o derecha.

En síntesis, los temas que más han interesado a los investigadores, al menos a partir de los trabajos recogidos en la B.D. "América Latina" sobre las transiciones a la democracia, son aquellos relacionados con las instituciones políticas y su funcionamiento, con los procesos electorales y con el desarrollo político. El interés por otros problemas de las democracias recientes como los niveles de renta, las políticas distributivas, las reformas económicas, las relaciones económicas internacionales, etc., fue bastante más secundario, si tomamos como indicadores los descriptores temáticos de los documentos. Menos aún han preocupado los aspectos relativos a los marcos socioculturales en los que los procesos de democratización han tenido lugar.

Más adelante volverán a tratarse los contenidos dominantes de los trabajos, analizando éstos por países. Ahora, parece interesante establecer algunas comparaciones con la producción bibliográfica sobre la transición española. 


\section{Encuentros y desencuentros con la bibliografía de la transición española ${ }^{7}$}

Se ha considerado interesante establecer algunas comparaciones entre el corpus bibliográfico estudiado en esta comunicación y la bibliografía publicada en revistas españolas sobre la transición política en España entre 1976 y 1991. A pesar de la diferencia en el tiempo de publicación, son dos conjuntos de información bastante comparables, pues los dos abarcan la producción de quince años, y los procesos de transición latinoamericanos fueron posteriores en sus inicios al español, de manera que la mirada de los investigadores sobre esos procesos puede ser comparable, sobre todo si no buscamos conclusiones fehacientes sino aproximaciones y rasgos comunes o diferenciales.

El segundo conjunto bibliográfico está compuesto por prácticamente el doble de documentos, 427 artículos de revistas españolas. Por ello para el estudio de los temas, en el primer conjunto la frecuencia mínima considerada ha sido 5, mientras que en este segundo conjunto ha sido 10 .

Los parámetros cuantitativos de la bibliografía sobre la transición española son los siguientes:

Número de documentos . . . . . . . . . . . . . . . 427

Número total de descriptores únicos . . . . . . . . . 1.232

Descriptores asignados a los documentos . . . . . . . 4.247

Media de descriptores por documento $\ldots \ldots \ldots \ldots \ldots 9,9$

Un análisis más profundo de las analogías y diferencias de los dos procesos y de su reflejo en la bibliografía requeriría un espacio superior al marco de esta comunicación. Por ello se considerarán sólo los contenidos generales dominantes, así como los temas más tratados.

\section{Contenidos generales}

Ámbito Transición española Transiciones latinoamericanas

\begin{tabular}{lrr}
\hline Descriptores "E" & $5,7 \%$ & $14,6 \%$ \\
Descripores "SC" & $18,7 \%$ & $12,7 \%$ \\
Descriptores "JP" & $64,2 \%$ & $46,2 \%$
\end{tabular}

7 Los datos sobre la bibliografía de la transición española han sido tomados de Román Román: Las revistas de Ciencias Sociales, fuente para el estudio de la Historia..., Capítulo III. Ver también Román Román, A.: "The spanish transition to democracy seen through the spanish database ISOC”. Scientometrics, 1994, 30 (1), págs. 201-212. 
Es claramente visible el predominio en los dos casos de los temas relativos al ámbito jurídico político y es también común la gran diferencia de interés entre estos últimos y los aspectos económicos y sociales, advirtiéndose aquí alguna diferencia notable: mucha más presencia de temas económicos en los estudios que tienen por objeto la transición democrática en los países latinoamericanos y mayor preocupación por los aspectos socioculturales en los autores de los trabajos sobre la transición española.

Para ver con más claridad cuáles son los aspectos más abordados en cada uno de los dos conjuntos bibliográficos, aportamos a continuación los diez términos más representativos de cada uno de los dos conjuntos estudiados y sus frecuencias absolutas:

Transición española

\begin{tabular}{llll}
\hline Franquismo & 74 & Partidos políticos & 57 \\
Democracia & 58 & Elecciones & 45 \\
Partidos políticos & 53 & Dictadura & 50 \\
Crisis económica & 43 & Fuerzas armadas & 33 \\
Elecciones legislativas & 43 & Crisis económica & 31 \\
Nacionalismo & 32 & Consolidación democ. & 28 \\
Terrorismo & 32 & Relaciones intern. & 27 \\
Ideología política & 25 & Sistema político & 25 \\
Reforma política & 24 & Política exterior & 23 \\
Política económica & 23 & Movimientos soc. & 19
\end{tabular}

Si se analizan un poco estas dos columnas, hay elementos comunes muy evidentes. En ambos corpus aparecen como elementos más estudiados los "partidos políticos", las "elecciones", la "democracia", en los dos casos la transición tiene lugar en un contexto de "crisis económica" reflejada en estas 10 primeras posiciones. La realidad de la "dictadura" o el "franquismo", que para el caso podemos considerar si no sinónimos, sí equivalentes, aparecen en posiciones muy dominantes, no podría ser de otra manera. Otros términos, de estos diez primeros, reflejan algunas par-

8 Los términos de búsqueda empleados para recuperar la bibliografía sobre la transición española fueron exclusivamente "transición política" o "transición democrática". Ver: Román Román: Las revistas de Ciencias Sociales... 
ticularidades de estos procesos a uno y otro lado del Atlántico: en España, el "nacionalismo" y el "terrorismo", en América Latina, la preocupación y la necesidad de apoyo de los países democráticos a los procesos de democratización, reflejados en los términos "relaciones internacionales" y "política exterior". Y, por último, hay en estas 10 primeras posiciones un reflejo simbólico de las preocupaciones de segundo nivel: en España, la "política económica", en América Latina, los "movimientos sociales", en línea con lo que se refleja a nivel más global en los datos de los apartados anteriores.

\section{Países abordados en los trabajos y temática por países}

\section{Países}

El 24\% de los documentos estudiados tratan de América Latina en general sin especificar países concretos o subregiones. Considerando las subregiones, América Central es la más tratada con un $21 \%$, sumando los documentos que tratan de cada país centroamericano en particular y los que hablan de la región en general.

En cuanto a los países en concreto, el más estudiado es Argentina (14\%), en segundo lugar Chile (11\%), le sigue Brasil (10\%), Uruguay (7\%), México (7\%) y Nicaragua y El Salvador con un 6\% cada uno. El resto de los países se encuentran casi todos presentes, aunque con un número de trabajos muy pequeño $(15 \%)$.

Esta distribución no causa demasiadas sorpresas a los que trabajamos habitualmente con documentación española sobre América Latina, especialmente la que trata de política, economía, sociología, etc. Si consideramos otros temas de estas disciplinas objeto de estudio de los americanistas españoles o no españoles que publican en España, encontraremos unas proporciones parecidas.

Existen unos países que concitan la atención de la investigación más que otros como es el caso de Argentina, Chile o Brasil. En el tema que nos ocupa, sólo desentona Mexico, ya que suele ser uno de los países mas tratados en las publicaciones americanistas españolas. En este caso, su escasa representación se debe, seguramente, a que la transición democrática de México tiene unas características especiales y se aparta, temporal y conceptualmente, de lo que es un proceso de transición "típico". 
El caso de los países del área centroamericana es de destacar por su amplia representación: recordemos que en esta región, la peculiaridad de unos procesos de transición que constituyeron en la práctica un paso de la guerra a la paz, suscitó mucha atención, así como el protagonismo de España en los procesos de negociación entre los sectores enfrentados (Esquipulas, Pactos de San José).

Un país ausente en este tema es Cuba, cuya problemática aparece frecuentemente en las publicaciones españolas. Obviamente en este caso está claro que el tema no le toca particularmente.

En cuanto al resto de los países cuya representación es bastante baja (Paraguay, Bolivia, Colombia, etc.) como hemos explicado, es un hecho que suele repetirse al analizar la documentación americanista española de ciencias sociales.

También se puede pensar que el hecho de que Argentina y Chile sean los países cuyos procesos de transición más han interesado en España, obedece a que dichos procesos son hasta cierto punto equiparables al proceso de democratización español.

\section{Análisis temático por países}

Dado que en la Base de Datos el sistema de indización por descriptores temáticos desciende a un nivel muy específico de análisis, hemos agrupado los términos de manera que pudiéramos obtener unas categorías temáticas más generales.

Con este procedimiento hemos creado 5 grupos temáticos:

Desarrollo político de la transición: en este apartado hemos agrupado a todos los descriptores que hacen referencia a los actores políticos y los factores coyunturales del proceso de transición a la democracia. En este grupo se encuentran términos como: "partidos políticos", "militares", "elecciones", "dictadura", etc.

Sistemas políticos: bajo esta denominación hemos integrado todos los descriptores que se refieren a conceptos que, más allá de la mera coyuntura política, caracterizan a los sistemas políticos de una forma más global y a los procesos que al márgen del mero hecho electoral afectan a las instituciones y a la política a largo plazo. Ejemplos de estos descriptores temáticos son: "presidencialismo", "reforma constitucional", "centralismo", "división de poderes", "sistema judicial", etc. 
Aspectos económicos: en este epígrafe se han reunido todos los descriptores de caracter económico como "desarrollo económico", "política económica", "neoliberalismo", "inversiones", "crisis económica", etc.

Dimensión internacional: agrupa descriptores como "relaciones internacionales", "coyuntura internacional", "integración regional", "ayuda exterior", etc.

Aspectos socioculturales: en este grupo temático hemos integrado todo aquello que se puede decir que NO es política, pero que tiene lugar en los procesos de transición, o sea los problemas y conflictos sociales, las repercusiones del proceso de transición en la sociedad civil, en los movimientos sociales, en la cultura. Ejemplos de estos descriptores son "derechos humanos", "desigualdades sociales", "opinion pública", "cambio social", "pobreza", "violencia", "iglesia católica", etc.

Temas específicos de cada país: integran este grupo los descriptores que se refieren a problemas y circunstancias propias del país en cuestión. Ejemplos: "fraude electoral" en el caso de México, "guerra civil", "guerrilla", "conflicto bélico" o "negociaciones de paz" en el caso de países centroamericanos.

\section{Temática de los documentos que tratan de América Latina en general}

Desarrollo político .................... 20\%

Aspectos económicos . . . . . . . . . . . . . . 20\%

Sistemas políticos .................... 26\%

Aspectos socioculturales ................... 17\%

Dimensión internacional $\ldots \ldots \ldots \ldots \ldots \ldots \ldots \ldots$

\section{Temática de los documentos por países concretos}

Arg. Bras. Chile Urug. Salv. Nica. Méx.

$\begin{array}{lrrrrrrr}\text { Desarrollo político } & 36 & 39 & 37 & 50 & 26 & 28 & 31 \\ \text { Sistemas políticos } & 27 & 26 & 20 & 19 & 16 & 14 & 32 \\ \text { Aspectos económicos } & 21 & 15 & 9 & 7 & 11 & 17 & 16 \\ \text { Aspectos socioculturales } & 11 & 19 & 21 & 15 & 10 & 10 & 12 \\ \text { Dimensión internacional } & 5 & - & 9 & 9 & 5 & 8 & - \\ \text { Temas específicos } & - & - & - & - & 30 & 23 & 10\end{array}$


Vemos que en lo que se refiere a los documentos que tratan de América Latina en general, sin detenerse en los países en concreto, la temática está bastante uniformemente repartida, siendo los aspectos políticos los más tratados y los socioculturales los que menos aparecen. Sin embargo, cuando analizamos la distribución temática por países concretos, la homogeneidad desaparece o al menos no está tan clara.

Los aspectos estrictamente políticos (coyunturales y no coyunturales) dominan con más de un 50\% del total de descriptores temáticos, sumando los dos grupos: "desarrollo político de la transición" y "sistemas políticos", mientras que los otros grupos temáticos son abordados con mucho menos peso. Existen algunas excepciones: por ejemplo, en el caso de Argentina, los aspectos económicos ocupan un lugar importante, por encima de la media, con un $21 \%$ de los descriptores, o el caso de Chile con un $21 \%$ también para el ambito sociocultural. También son excepción El Salvador, Nicaragua y México, en relación al alto grado de aparición de temas que se refieren a cuestiones particulares de estos países, representados por porcentajes altos de descriptores de los que hemos llamado "específicos".

Este hecho plantea algunos interrogantes: ¿por qué cuando se analizan los procesos de transición concretos de los países se aborda sobre todo el aspecto político, dejando de lado, o al menos relegando a un segundo plano la problemática económica, social y cultural de los países en cuestión?

Sólo podemos apuntar, especulativamente, algunas razones de estos desequilibrios, ya que con los datos que tenemos tampoco es posible establecer una relación directa causa-efecto. Los aspectos económicos, sociales y culturales, así como los problemas específicos de cada país y de cada proceso de transición posiblemente requieren un nivel de estudio y de análisis en profundidad que no es el que encontramos en los documentos estudiados. Los temas estrictamente políticos, especialmente los relacionados con la coyuntura, son los que aparecen en una "primera mirada", son, diríamos, los más evidentes de una transición politica. Descender a analizar lo que ocurre "detras de la cortina política", internarse en el desarrollo de la sociedad civil, en lo que late al márgen de los partidos, los parlamentos, los ejércitos o los centros de poder, implica realizar un estudio detenido y profundo que quizás esté aún por hacer. Es probable que esto sea una cuestión de etapas, de tiempos, es decir que en un primer momento el interés se centra en lo político y la reflexión sobre otros aspectos (internacional, social, cultural, etc.) de un proceso de transición sea algo que inevitablemente venga más tarde, una vez aplacado el escenario político, con más perspec- 
tiva. El interés demostrado por problemáticas particulares ("temas específicos") en el caso de El Salvador, Nicaragua y México se debe probablemente más a la particularidad del propio proceso de democratización que a un análisis más pormenorizado de dichos países.

Por último, en cuanto al desfase que aparece en los temas referentes a la dimensión internacional de los procesos de transición, segun se trate de América Latina como un todo o de cada país en particular (18\% en el primer caso y $9 \%$ de media en el segundo), lo que podemos deducir es que el interés se ha centrado en las relaciones internacionales a nivel de "bloques", es decir, relaciones de la Comunidad Europea con la América Latina democrática, posturas de las distintas administraciones norteamericanas respecto de los procesos de democratización latinoamericanos, etc. 